\title{
A THEOREM ON GENERAL RECURSIVE FUNCTIONS
}

\author{
SHIH-CHAO LIU
}

The purpose of this note is to prove the following theorem.

THEOREM. Every general recursive function $f(n)$ is expressible in the form $f(n)=g\left(2^{n}\right)$ for some function $g$ defined by the following schema

$$
\begin{aligned}
g(0) & =0, \\
g\left(n^{\prime}\right) & =\theta\left(n^{\prime}, g\left(\delta\left(n^{\prime}\right)\right)\right)
\end{aligned}
$$

where $\theta$ and $\delta$ are primitive recursive and $\delta\left(n^{\prime}\right)<n^{\prime}$ in some primitive recursive well-ordering of the natural numbers of order type $\omega$.

This theorem is an improved version of the result obtained by Myhill [1] as well as a similar one obtained by Routledge [2, Theorem 4]. As functions of one variable are concerned, the foregoing theorem is the same as Routledge's except that the well-ordering く referred to in this note is primitive recursive while it is defined by general recursive processes in Routledge's paper (see [2, Theorem 2]). Myhill's statement is, however, not sufficiently explicit. A more explicit statement of his result was supplied by Kleene in a letter to the author. The theorem in this note differs from Kleene's formulation only in that Kleene expressed $f(n)$ as $U\left(g\left(2^{n}\right)\right)$ while here the function $U$ is not used. This leads to the result $f(n)=g\left(2^{n}\right)$, which is, according to Routledge (see $[2, \S \S 8$ and 9$]$ ), the best possible in the sense that we can no longer omit the function $2^{n}$ and therefore can not simply equate $f(n)$ and $g(n)$.

Proof of the TheOREM. Let $f(n)$ be any given general recursive function. According to a theorem by Kleene [3, p. 288] there are two primitive recursive functions $K(n)$ and $T(x, y)$ such that

$$
(x)(E y)(T(x, y)=0) \text { and } f(n)=K(\mu y(T(n, y)=0))
$$

where $\mu y(T(n, y)=0)$ is the least $y$ such that $T(n, y)=0$.

In terms of $K(n)$ and $T(x, y)$ we define the function $g(n)$ in the following manner:

$$
\begin{aligned}
& g(0)=0, \\
& g\left(n^{\prime}\right)=\left\{\begin{array}{l}
g\left(2^{x} \cdot 3^{y+1}\right), \text { if } n^{\prime}=2^{x} \cdot 3^{y} \text { and } T(x, i) \neq 0 \text { for } i<y+1, \\
K(y), \text { if } n^{\prime}=2^{x} \cdot 3^{y} \text { and } T(x, y)=0 \text { but } T(x, i) \neq 0, \text { if } i<y, \\
0, \text { otherwise. }
\end{array}\right.
\end{aligned}
$$

Received by the editors April 23, 1959. 
Consequently we have, for every $n$, $g\left(2^{n}\right)=g\left(2^{n} \cdot 3^{1}\right)=g\left(2^{n} \cdot 3^{2}\right)=\cdots=g\left(2^{n} \cdot 3^{a_{n}-1}\right)=g\left(2^{n} \cdot 3^{q_{n}}\right)=K\left(q_{n}\right)$ where $q_{n}=\mu y(T(n, y)=0)$. By the second expression of (1) we then have

$$
f(n)=g\left(2^{n}\right),
$$

In order to reformulate this definition of $g$ we first introduce the following notations:

$A(n)=0 \leftrightarrow n$ can be written in the form $2^{x} \cdot 3^{y}$ and $T(x, i) \neq 0$ for $i<y+1$.

$B(n)=0 \leftrightarrow n$ can be written in the form $2^{x} \cdot 3^{y}$ and $T(x, y)=0$ and $T(x, i) \neq 0$, if $i<y$.

Following the notations in Kleene's book [3] we can show that ${ }^{1}$

$$
\begin{aligned}
A(n)=0 \leftrightarrow n=2^{(n)_{0}} \cdot 3^{(n)_{1}} & \& \prod_{i<(n)_{1}+1} T\left((n)_{0}, i\right) \neq 0, \\
B(n)=0 \leftrightarrow n=2^{(n)_{0}} \cdot 3^{(n)_{1}} & \& T\left((n)_{0},(n)_{1}\right)=0, \\
\& & \prod_{i<(n)_{1}} T\left((n)_{0}, i\right) \neq 0 .
\end{aligned}
$$

We see that $A(n)$ and $B(n)$ are primitive recursive functions. In terms of these and some other primitive recursive functions ${ }^{2}$ we can reformulate the definition of $g(n)$ as follows:

$$
\begin{aligned}
g(0) & =0, \\
g\left(n^{\prime}\right) & =\bar{s} \bar{g}\left(A\left(n^{\prime}\right)\right) \cdot g\left(\bar{s} \bar{g}\left(A\left(n^{\prime}\right)\right) \cdot 2^{\left(n^{\prime}\right)_{0}} \cdot 3^{\left(n^{\prime}\right)_{1}+1}\right)+\bar{s} \bar{g}\left(B\left(n^{\prime}\right)\right) \cdot K\left(\left(n^{\prime}\right)_{1}\right) .
\end{aligned}
$$

Let $\theta\left(n^{\prime}, x\right)$ stand for $\bar{s} \bar{g}\left(A\left(n^{\prime}\right)\right) \cdot x+\bar{s} \bar{g}\left(B\left(n^{\prime}\right)\right) \cdot K\left(\left(n^{\prime}\right)_{1}\right)$ and $\delta\left(n^{\prime}\right)$ stand for $\bar{s} \bar{g}\left(A\left(n^{\prime}\right)\right) \cdot 2^{\left(n^{\prime}\right) 0} \cdot 3^{\left(n^{\prime}\right) 1+1}$. Then the definition of $g$ can be written as

$$
\begin{aligned}
g(0) & =0, \\
g\left(n^{\prime}\right) & =\theta\left(n^{\prime}, g\left(\delta\left(n^{\prime}\right)\right)\right)
\end{aligned}
$$

where $\theta(n, x)$ and $\delta(n)$ are both primitive recursive.

To complete the proof it remains only to find a primitive recursive well-ordering $\prec$ of the natural numbers of type $\omega$ and then show that $\delta\left(n^{\prime}\right) \prec n^{\prime}$ for every $n$. We first introduce the notations ${ }^{3} x \in C$ and $H(x)$

1 We note, in particular, that $(0)_{i}=0,\left(2^{x} \cdot 3^{y}\right)_{0}=x$ and $\left(2^{x} \cdot 3^{y}\right)_{1}=y$; for any function $\psi, \prod_{i<z} \psi(i)=1$, if $z=0$.

$2 \overrightarrow{s g}(n)=0$, if $n \neq 0 ; \vec{s} g(n)=1$, if $n=0$.

${ }^{3}$ See $\left[3\right.$, p. 225]. For any predicate $R(x), \mu t_{t<z} R(t)$ is the least $t<z$ such that $R(t)$, if $(E t)_{t<z} R(t)$; otherwise, $z$. 
defined as follows:

$$
\begin{array}{r}
x \in C \leftrightarrow A(x)=0 \vee B(x)=0, \\
H(x)=x-\mu t_{t<x}((x-t) \in C) .
\end{array}
$$

$H(x)$ is primitive recursive and has the following properties:

1. $H(x)=x$, if and only if $x=0$ or $x \in C$;

2. $H(x) \leqq x$ and $H\left(x^{\prime}\right) \in C$;

3. $H\left(x^{\prime}\right)$ is the number $y$ nearest to $x^{\prime}$ such that $y \leqq x^{\prime}$ and $y \in C$. by

In terms of $H(x)$ a primitive recursive binary relation $\prec$ is defined

$$
\begin{aligned}
x<y \leftrightarrow(H(x) & =H(y) \& x<y) \vee\left((H(x))_{0}<(H(y))_{0}\right) \vee\left((H(x))_{0}\right. \\
& \left.=(H(y))_{0} \& H(x)>H(y)\right) .
\end{aligned}
$$

According to this order $\prec$ all the natural numbers are arranged in a linear order as follows:

$$
\begin{aligned}
0 & <2^{0} \cdot 3^{q_{0}}<\cdots<2^{0} \cdot 3^{0}<\cdots<2^{1} \cdot 3^{q_{1}}<\cdots<2^{1} \cdot 3^{0}<\cdots \\
& <2^{i} \cdot 3^{j}<2^{i} \cdot 3^{i}+1<2^{i} \cdot 3^{j}+2<\cdots<2^{i} \cdot 3^{j}+\sigma(i, j)<2^{u} \cdot 3^{v} \\
& <\cdots
\end{aligned}
$$

where $2^{i} \cdot 3^{i} \in C ; q_{n}=\mu y(T(n, y)=0) ; u=i$ and $v=j-1$, if $j \neq 0$; but $u=i+1$ and $v=q_{i+1}$, if $j=0 ; 2^{i} \cdot 3^{j}+\sigma(i, j)$ is the greatest number $y$ such that $H(y)=2^{i} \cdot 3^{j}$. The value of $2^{i} \cdot 3^{j}+\sigma(i, j)$ is a finite number and can be calculated in the following manner. Let $2^{i} \cdot 3^{i}+1=k$. Then $2^{i} \cdot 3^{j}<k<2^{k}$ and $2^{k}=2^{k} \cdot 3^{0} \in C$. It must be that $H(z) \neq 2^{i} \cdot 3^{j}$ for every $z>2^{k}$. For, otherwise, there would be a $z>2^{k}$ such that $H(z)=2^{i} \cdot 3^{j}$. Then according to the third property of $H(x), 2^{i} \cdot 3^{j}$ would be the number $y$ nearest to $z$ such that $y \leqq z$ and $y \in C$. But this contradicts the fact that $2^{k} \in C$ and $2^{i} \cdot 3^{i}<2^{k}<z$. Thus we have

$$
2^{i} \cdot 3^{j}+\sigma(i, j)=2^{k} \dot{-} \mu t_{t<2^{k}}\left(H\left(2^{k}-t\right)=2^{i} \cdot 3^{j}\right) .
$$

The above ordering shows that $x<y$ is a primitive recursive wellordering of the natural numbers of type $\omega$ with 0 as the first element.

Now let us consider the function

$$
\delta\left(n^{\prime}\right)=\bar{s} \bar{g}\left(A\left(n^{\prime}\right)\right) \cdot 2^{\left(n^{\prime}\right) 0} \cdot 3^{\left(n^{\prime}\right) 1+1}
$$

which appears in the third definition of $g$. In case $A\left(n^{\prime}\right)=0$ we

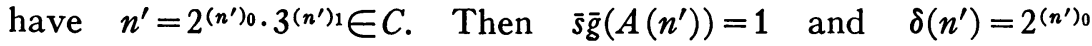
$\cdot 3^{\left(n^{\prime}\right) 1+1}$. $\delta\left(n^{\prime}\right)$ must belong to $C$. By the first property of $H(x)$, we have $H\left(n^{\prime}\right)=n^{\prime}=2^{\left(n^{\prime}\right)_{0} \cdot 3^{\left(n^{\prime}\right)_{1}}}$ and $H\left(\delta\left(n^{\prime}\right)\right)=\delta\left(n^{\prime}\right)=2^{\left(n^{\prime}\right)_{0}} \cdot 3^{\left(n^{\prime}\right)_{1}+1}$. Consequently,

$$
\left(H\left(\delta\left(n^{\prime}\right)\right)\right)_{0}=\left(H\left(n^{\prime}\right)\right)_{0}=\left(n^{\prime}\right)_{0} \& H\left(\delta\left(n^{\prime}\right)\right)>H\left(n^{\prime}\right) .
$$


According to the definition of $\prec$ we have $\delta\left(n^{\prime}\right)<n^{\prime}$. In case $A\left(n^{\prime}\right) \neq 0$ we have $\bar{s} \bar{g}\left(A\left(n^{\prime}\right)\right)=0$. Then $\delta\left(n^{\prime}\right)=0$. Since 0 is the first element in the ordering $\prec$ we also have $\delta\left(n^{\prime}\right) \prec n^{\prime}$. Thus in any case $\delta\left(n^{\prime}\right) \prec n^{\prime}$. This shows that the definition of $g(n)$ is an ordinal recursion schema with respect to $\prec$. This completes the proof of the theorem.

\section{REFERENCES}

1. J. Myhill, A stumbling block in constructive mathematics, J. Symb. Logic vol. 18 (1953) pp. 190-191.

2. N. A. Routledge, Ordinal recursion, Proc. Cambridge Philos. Soc. vol. 49 (1953) pp. 175-182.

3. S. C. Kleene, Introduction to metamathematics, New York and Toronto, Van Nostrand, 1952.

Institute of Mathematics, Academia Sinica,

Taiwan, China 